\title{
Coding Telemedicine Visits for Proper Reimbursement
}

\author{
Gary N. Gross ${ }^{1}$
}

Accepted: 4 September 2020 / Published online: 2 October 2020

(C) Springer Science+Business Media, LLC, part of Springer Nature 2020

\begin{abstract}
Purpose of Review Coding for patient visits and monitoring via telehealth have expanded over the past years with a wide acceptance of telemedicine as a consequence of the coronavirus pandemic. Coding topics of interest to the allergist/ immunologist in regard to services provided via telemedicine will be of increasing importance in the coming years.

Recent Findings CPT coding for telephone as well as synchronous face-to-face telehealth visits has changed over the past few years. With the need for distancing and patient protection during the coronavirus pandemic, telehealth services have increased dramatically. The introduction of newer devices to remotely monitor patients will increase and be incorporated into patient care. Summary This review will summarize current codes available for designating what services have been provided. The area of telemedicine is changing and will continue to evolve as other platforms for visits are designed and other methods of monitoring patients become available. Coding for these services will be an ongoing need for the provider.
\end{abstract}

Keywords Coding $\cdot$ Telemedicine $\cdot$ Telehealth $\cdot$ Remote monitoring

\section{Introduction}

Current procedural terminology (CPT) has recognized the need for designations of procedures done using technology. Although initial codes focused primarily on telephone visits, in 2017, CPT recognized a new place-of-service (POS) code designating "The location where health services and health related services are provided or received, through a telecommunication system." This POS " 02 " was a step forward in awareness of the need for distant patient encounters and procedures. Further codes for both monitoring and evaluating via telehealth will be discussed. Table 1 lists current CPT codes available for designating services provided.

\section{Telehealth Coding}

Two words must be remembered whenever coding is discussed. The two words, "it depends," define the lack of

This article is part of the Topical Collection on Telemedicine and Technology

Gary N. Gross

gary.gross@daac-prc.com

1 Internal Medicine, Southwestern Medical Center, University of Texas, Dallas, TX, USA consistency in coding throughout the industry. Coding is generally driven by The Centers for Medicare \& Medicaid Services (CMS) and CPT (although they do not always align). CPT codes exist for procedures, but some carriers may not recognize or reimburse for the codes [2]. Some carriers may create their own limits on reimbursing for codes, arbitrarily considering procedures bundled with evaluation and maintenance (E\&M) visits. Codes may be paid for certain disease states but not for others. Insurers vary with regard to expectations of what place-of-service to use or how to bill for some procedures. New modifiers for telehealth visits further complicate billing. The modifiers -GT and - 95 are used by some carriers for telehealth visits and vary depending on the insurer. Similarly, place of service may be either " 02 ," the telecommunication POS mentioned above, or remain "11" which designates the office location. Therefore, one must be flexible and informed. Keeping track of each carrier's latest provider information and appealing denials with alternative codes may be necessary.

As with conventional patient encounters, documentation is key. For telehealth visits, there is also the need to document the patient's consent for the encounter via telehealth. Most of the telehealth codes are for providers who could bill for evaluation and management $(\mathrm{E} / \mathrm{M})$ services such as physicians, physician assistants (PAs), and nurse practitioners (NPs). These providers are considered qualified healthcare providers (QHP). 
Table 1 Current CPT codes [1]

Code Description

Telehealth visits (face-to-face)

Audio/visual visit between a patient and clinician for evaluation and management (E\&M) New patient level one code (99201) has been deleted.

CPT code 99202-99205 Office or other outpatient visit for the evaluation and management of a new patient

CPT code 99212-99215 Office or other outpatient visit for the evaluation and management of an established patient

Check with your payer to determine the appropriate Place of Service (POS) code for your telehealth visits.

Telephone evaluation and management service (non-face-to-face)

Evaluation and management visits via audio-only (telephone) communications

CPT code $99441 \quad$ Telephone evaluation and management service by a physician or other qualified healthcare professional (QHP) who may report evaluation and management services provided to an established patient, parent, or guardian not originating from a related E/M service provided within the previous 7 days nor leading to an E/M service or procedure within the next $24 \mathrm{~h}$ or soonest available appointment; 5-10 min of medical discussion; these calls are initiated by the patient or guardian of patient.

CPT code 99442

11-20 min of medical discussion

CPT code 99443

21-30 min of medical discussion

\section{Remote patient monitoring}

Collecting and interpreting physiologic data digitally stored and/or transmitted by the patient and/or caregiver to the physician or QHP.

CPT code 99453

Remote monitoring of physiologic parameter (s) (e.g. pulse oximetry, respiratory flow rate), initial; set-up and patient education on use of equipment.

CPT code 99454

Device (s) supply with daily recording (s) or programmed alert (s) transmission, each 30 days. (Initial collection, transmission, and report/ summary services to the clinician managing the patient); each 30 days (provided monitoring occurs during at least 16 days during the 30 -day period)

CPT code 99457

Remote physiologic monitoring treatment management services, clinical staff/physician/other QHP time in a calendar month requiring interactive communication with the patient/caregiver during the month; first $20 \mathrm{~min}$

CPT code 99458

Each additional $20 \mathrm{~min}$ (list separately in addition to code for primary procedure)

CPT code 99091

Collection and interpretation of physiologic data (e.g., blood pressure, glucose monitoring) digitally stored and/or transmitted by the patient and/ or caregiver to the physician or other QHP, qualified by education, training, licensure/regulation (when applicable) requiring a minimum of 30 min each time, each 30 days)

CPT code 94014

Patient initiated remote spirometry, transmissions of tracings and review and interpretation by a physician or QHP.

CPT code 94015

Patient initiated remote spirometry, does not include review and interpretation by a physician or other QHP

CPT code 94016

Patient initiated remote spirometry, review and interpretation by provider

Online digital evaluation and management services

CPT code 99421

CPT code 99422

CPT code 99423

For providers who can bill EM codes, online digital evaluation and management service, for an established patient, for up to 7 days, cumulative time during the 7 days; $5-10 \mathrm{~min}$

For providers who can bill EM codes; $11-20$ min

For providers who can bill EM codes; 21 or more minutes

CPT code 98970 (HCPCS code For providers who cannot bill EM codes, qualified non-physician healthcare professional online digital assessment and management, for an established patient, for up to 7 days, cumulative time during the 7 days; 5-10 min

CPT code 98971 (HCPCS code For providers who cannot bill EM codes; 11-20 min

G2062)

CPT code 98972 (HCPCS code For providers who cannot bill EM codes; 21 or more minutes

HCPCS code G2012

Brief communication technology-based service, e.g. virtual check-in, by a physician or other QHP who can report evaluation and management services, provided to an established patient, not originating from a related E/M service provided within the previous 7 days nor leading to an E/M service or procedure within the next $24 \mathrm{~h}$ or soonest available appointment; 5-10 min of medical discussion

HCPCS code G2010

Remote evaluation of recorded video and/or images submitted by an established patient (e.g., store and forward), including interpretation with follow-up with the patient within 24 business hours, not originating from a related E/M service provided within the previous 7 days nor leading to an $\mathrm{E} / \mathrm{M}$ service or procedure within the next $24 \mathrm{~h}$ or soonest available appointment.; 5-19 min of medical discussion

\section{Interprofessional telephone/internet/electronic health record consultations}

CPT code 99446

Consultation when there is an interprofessional electronic consultation regarding assessment and management of a patient who is not seen face-to-face by the consulting provider. The patient or family must give verbal consent (documented in the record) for the consult 
Table 1 (continued)

\begin{tabular}{ll}
\hline Code & Description \\
\hline & $5-10$ min of consultative discussion/review \\
CPT code 99447 & $11-20 \mathrm{~min}$ \\
CPT code 99448 & $21-30 \mathrm{~min}$ \\
CPT code 99449 & $31 \mathrm{~min}$ or more \\
CPT code 99451 & 5 min or more time but does not require that more than $50 \%$ of the time be consultative time as opposed to data review. Requires \\
& a written report \\
CPT code 99452 & Time spent in preparing the consult and/or time communicating with the consultant for 16 min or more time
\end{tabular}

\section{Non-face-to-face Telehealth Patient Visits}

Telephone Services (99441-99443) These codes are non-faceto-face E/M services used by QHP. They are designed for telephone calls initiated by an established patient and have certain restrictions. If the call includes the decision to see the patient in the next $24 \mathrm{~h}$ or next available urgent appointment, it cannot be billed. Similarly, if the call refers to an E/M service reported by the QHP within the past 7 days, the telephone codes cannot be used. Thus, these calls are initiated by the patient or guardian of the patient and stand apart from other $\mathrm{E} / \mathrm{M}$ visits as described.

99441 - 5-10 min of medical discussion 99442 - 11-20 min of medical discussion 99442 - 11-30 min of medical discussion

Example An established patient, who has not been seen in the past month, calls the office because of a recent ant bite. The patient wants to speak to the physician since the physician also treats the son for anaphylaxis to wasps and the patient is concerned. The physician talks to the patient about the kinds of reactions that might occur and notifies him of what symptoms he should be aware of. Out of an abundance of caution, the physician reminds the patient about using an epinephrine autoinjector. The conversation takes $25 \mathrm{~min}$. The staff calls in the autoinjector to the pharmacy and is on the phone for 15 min waiting for the pharmacist.

The patient is billed 99443 for the physician time. The staff time would not enter into the total time. The note in the chart would document that the visit was via telephone and that the patient called the clinic about the problem. The discussion would be documented and the note would indicate the patient had not been seen and no E/M visit was anticipated. The note would also indicate that $25 \mathrm{~min}$ was spent in discussion.

Online Digital Evaluation and Management Services (9942199423) These codes are electronic communication codes. The problem may be new to the physician or QHP but the patient must be established. These services are patient-initiated through HIPAA-compliant secure platforms or portals.

These services include evaluation, assessment, and management of the patient.

These services are reported once during a 7-day period and therefore time is cumulative.

The time includes (1) review of the initial inquiry, (2) review of patient records or data pertinent to assessment of the patient's problem, (3) interaction with clinical staff focused on the patient's problem and development of management plans, (4) physician or other QHP generation of prescriptions or ordering of tests, and (5) subsequent communication with the patient through online, telephone, email, or other digitally supported communication, which does not otherwise represent a separately reported E/M service.

These services require permanent documentation storage (electronic or hard copy) of the encounter.

If within 7 days of the initial patient-initiated contact a separate $\mathrm{E} / \mathrm{M}$ visit (in person or synchronous telemedicine) occurs, then the Online Digital visit is not billed but the time is incorporated into the subsequent $\mathrm{E} / \mathrm{M}$ visit. If the Online Digital visit is initiated within 7 days of a previous $\mathrm{E} / \mathrm{M}$ visit for the same or related problem, the Online Digital visit is not reported. If a new or different problem is being addressed in the Online Digital visit, then the visit is billable and should be reported.

99421-5-10 min (over a 7-day period)

99422-11-20 min (over a 7-day period)

99423-21 or more minutes (over a 7-day period)

Remember that only physician or other QHP time is used in the calculation. Staff time is not included.

Example An established patient who was seen 3 days ago for allergic rhinitis wakes up with hives. She uses the practice's HIPAA compliant portal to message her doctor about the hives. The PA responds to the message and gathers information about the hives, the patient's activities, and ultimately prescribes an antihistamine. The encounter takes $10 \mathrm{~min}$. 
Two days later, the patient messages again saying the hives are better but not gone. She wants stronger medicine. The PA responds to the message and offers to prescribe a short course of corticosteroids. The PA describes the possible side effects of the steroids and also tells the patient what should be done if the hives do not clear. The PA spends 12 min with the encounter. The patient does not call back and does not come to the office for the hives. The PA bills the patient 99423 since the sum of the two encounters was $22 \mathrm{~min}$ within a 7-day period and the hives were not related to the allergic rhinitis the patient had been seen for 3 days before the hives.

The chart would document that the patient contacted the clinic for a new problem. All time spent by the PA would be documented to support the total time billed. It would be documented that no $\mathrm{E} / \mathrm{M}$ visit was anticipated for this new problem.

\section{G Codes}

Healthcare Common Procedure Coding System (HCPCS) have 2 levels of commonly used codes. Level 1 codes are CPT codes and level 2 codes are alphanumeric codes. One group of HCPCS codes are "G codes." The G codes are used to identify professional healthcare procedures and services that would otherwise be coded in CPT but for which there are no CPT codes. Two "G codes" are relevant to telehealth and do not yet have matching CPT codes [3].

G2010 was in the 2019 physician fee schedule and is used for remote evaluation of established patient's submitted videos or still images. The purpose of the evaluation is to determine whether or not an $\mathrm{E} / \mathrm{M}$ visit is necessary. It may be billed if the evaluation does not lead to an $\mathrm{E} / \mathrm{M}$ visit and does not occur within 7 days of a previous $\mathrm{E} / \mathrm{M}$ visit. To bill for the evaluation, the physician or other QHP must evaluate the image within 24 business hours and follow-up with the patient in the form of a 5-10 min discussion with the patient.

Example An established patient develops a rash and is uncertain about its cause. The patient sends the physician a picture of the rash. The physician evaluates the photo and determines it is a hive. The physician calls the patient and tells him that these are common and if they last more than 6 weeks or get worse he can check back, but that he does not need to have an $\mathrm{E} / \mathrm{M}$ visit.

Documentation of this remote evaluation would include the picture in the chart and the provider's note that the picture was viewed and that no visit would be necessary unless the hives lasted more than 6 weeks. The presumed diagnosis of acute urticaria would also be included.
G2012 was also included in the 2019 physician fee schedule. It has been referred to as a "virtual check-in." It is considered to be a call or video check in to see if an E/M visit is needed. Similar to some other e-codes, it cannot be billed if there was a related $\mathrm{E} / \mathrm{M}$ service within the previous 7 days or it leads to an E/M visit within the next $24 \mathrm{~h}$ or soonest available appointment. The code is used for established patients having direct interaction with the billing practitioner (not the staff). The service must be medically reasonable and necessary but there is no limitation on frequency. The code assumes $5-10 \mathrm{~min}$ of medical discussion.

Example An established patient calls the nurse practitioner and describes a large, local reaction they have from a mosquito bite. The patient wants to know if they need to come in or go to the ER. The nurse practitioner informs the patient about the type of reaction and tells the patient they only need to come in if they have trouble breathing or if the reaction spreads. The patient is reassured and watches the reaction as it gradually goes away. The practitioner can bill G2012.

The documentation for this virtual check in would include the main points of discussion including the bite and the likely diagnosis as well as the $5+$ min the provider is on the platform talking with the patient.

\section{Face-to-face Telehealth Patient Visits}

The Centers for Medicare \& Medicaid Services (CMS) defines telehealth services to include those services that require a face-to-face meeting with the patient. These are visits commonly considered "office visits" but delivered via synchronous audio and video contact with the patient. The usual E/ M visit codes (99201-99215) would apply.

Prior to 2021, these E/M visits level of service was determined by history, physical exam, and medical decisionmaking as documented in the CPT book each year. If more than $50 \%$ of the face-to-face time with the patient and/or family was used in counseling and/or coordination of care, time becomes the key factor in determining level of service.

Beginning with CPT 2021, time alone may be used to select the appropriate level for the office or other outpatient E/M services codes (99202, 99203, 99204, 99205, 99212, 99213, 99214, 99215). This "time" requires a face-to-face encounter with the physician or other QHP. Time spent with staff such as registering in the office or making future appointments is not used in the calculation of time. Also, note that the new patient level one code (99201) has been deleted.

Medical decision-making (MDM) includes establishing diagnoses, assessing the status of a condition, and/or selecting a management option. Beginning in 2021, MDM may be used independently in establishing a level of service (without consideration of history or physical exam as was required 
previously). These changes were designed to reduce duplication and unnecessary, repetitious documentation, and should also make coding for telehealth $\mathrm{E} / \mathrm{M}$ visits easier.

The telemedicine $\mathrm{E} / \mathrm{M}$ visits are coded just as an in-office $\mathrm{E} / \mathrm{M}$ visit would be but possibly with the addition of a modifier or a different place of service code depending on the insurance company. Some provisions for telemedicine have been waived during the pandemic to allow more patients access to medical care and to avoid exposure to others in waiting rooms and offices. The telemedicine waivers include evaluation of new patients via telehealth, beneficiaries living in any geographic area and accessing telemedicine from their homes, and use of smartphones and audio only connections for some services. Whether all these waivers will remain in place following the pandemic is unknown.

Example An established patient calls the office to set up an appointment and is offered a telemedicine option. The patient finds this attractive since it will save him time in traffic and reduce his time away from work. It is for a follow-up to see how he is doing after starting immunotherapy a month ago. The patient signs into the doctor's telehealth platform and gives verbal consent for the visit. They discuss symptoms, reactions to injections, medications, and concerns of the patient regarding future injections if he goes on vacation. The face-to-face time with the physician is $22 \mathrm{~min}$ and the code billed is 99213 (less than the minutes currently typical for 99214 and within the 20-29 min designated for 2021).

These telemedicine visits will require documentation similar to in-person visits. They will include the notation that the patient consents to the telehealth visit. Since the visits for new patients require physical examinations, the best way to document and bill these visits will be based on time. Until 2021, the notation that over $50 \%$ of the time with the patient was related to counseling and/or coordination of care is also needed. For follow-up visits before 2021, only two major components of the E/M visit are necessary, so history and medical decisionmaking with documentation could be used. It may be easier since most telemedicine visits are largely counseling and coordination of care, to base these encounters on total time also and indicate that greater than 50\% was devoted to counseling/ coordination of care. Typical documentation will include the consent for the visit, the discussion with the patient, the differential diagnosis, the plan of care, and the total "face-toface" time spent on the visit. The further notation that $>50 \%$ of the time was related to counseling and coordination of care (assuming it was) should also be documented.

\section{Remote Monitoring}

In addition to patient encounters whether non-face-to-face or face-to-face, the allergist/immunologist may also do remote monitoring of the patient. The 2020 CPT book lists the following codes for remote patient monitoring (RPM). Although some requirements for telehealth services have been modified during the pandemic, RPM services have never been limited by geography to rural or medically underserved areas, nor is there any "originating site" restriction for RPM services. In fact, RPM services can be provided anywhere the patient is located, including at the patient's home.

99453 Remote monitoring of physiologic parameter(s) (e.g., weight, blood pressure, pulse oximetry, respiratory flow rate), initial; set-up and patient education on use of equipment

99454 Device(s) supply with daily recording(s) or programmed alert(s) transmission, each 30 days (provided monitoring occurs at least 16 days during the 30-day period)

99457 Remote physiologic monitoring treatment management services, clinical staff/physician/other qualified healthcare professional time in a calendar month requiring interactive communication with the patient/caregiver during the month; first $20 \mathrm{~min}$.

99458 Each additional 20 min

As more devices become available to monitor asthma and other diseases treated by the allergist/immunologist, these codes will become more widely used just as blood pressure monitoring and diabetes monitoring are today.

Example An established patient is in the office and has poorly controlled, moderately severe persistent asthma. You provide the patient with a home spirometer that will transmit the FEV1 and FVC to your office. The patient is instructed on how to set up and use the device. He provides data via the portal for 20 days of the next month and you and your staff retrieve the data and analyze it. The time involved in retrieving and analyzing the information is $18 \mathrm{~min}$. You modify the patient's treatment program and describe the new treatment program during a telemedicine visit.

Billing would be 99453, 99454, and 99457 (each one unit). The E/M visit would be billed based on the time spent with the patient in describing the new treatment plan.

The documentation for these services might include a statement such as "we have provided this patient a remote spirometer and taught the patient its proper use. The patient has used it and transmitted information to us 20 days this month and the staff and I have spent 18 min total in the monitoring and responding to this patient in regard to asthma management based on results of the information transmitted."

An older CPT code used for remote patient monitoring is 99091. This older code requires $30 \mathrm{~min}$ to bill based on a 30day period. It is also limited to physicians and QHPs. There must be a face-to-face visit within 1 year and consent must be 
given and documented. The platform used must both collect and transmit data in real time or near real time to be eligible.

Another set of spirometry codes $(94014,94015,94016)$ relate to patient-initiated remote spirometry, transmission of tracings, and review and interpretation by a physician or QHP. The second code (94015) does not include review and interpretation by a physician or other QHP whereas the third code (94016) is the review and interpretation by the provider. 94014 is an inclusive code of the latter two.

\section{Interprofessional Telephone/Internet/Electronic Health Record Consultations}

Codes 99446, 99447, 99448, 99449, and 99451/99452 are used to report a consultation when there is an interprofessional electronic consultation regarding assessment and management of a patient who is not seen face-to-face by the consulting provider. The patient may be a new patient to the consultant or an established patient with a new problem. The patient should not have been seen by the consultant for a face-toface encounter in the past 14 days. Similarly, there should not be a transfer of care or a face-to-face encounter within the following 14 days of the consultation. Greater than $50 \%$ of the time for service must be devoted to the verbal or internet discussion. These codes should not be reported more than once within a 7-day interval. The consulting provider delivers a written or verbal report to the patient's treating provider. The patient or family must give verbal consent (documented in the record) for the consult.

99446 reported by the consulting provider for 5-10 $\mathrm{min}$ of consultative discussion/review

99447 11-20 min

99448 21-30 min

$9944931 \mathrm{~min}$ or more

Code 99451 is reported by the consultant for 5 min or more time but does not require that more than $50 \%$ of the time be consultative time as opposed to data review. Furthermore, 99451 requires a written report.

Code 99452 is billed by the treating/requesting provider. This code is for time spent in preparing the consult and/or time communicating with the consultant for $16 \mathrm{~min}$ or more time.

\section{Conclusions}

Telemedicine will continue to be a significant part of the allergy/immunology practice even after the pandemic. Both
Medicare and commercial insurance companies have made special provisions for telehealth during the pandemic in order to make medical care more readily available for patients who are concerned about their symptoms and also concerned about possible exposure to illness in a healthcare facility. Such provisions as allowing telephone calls (without video) to be sufficient for a "face-to-face" telemedicine visit for patients who do not have access to computers or other means of communicating via video connections will probably not continue after the pandemic [3, 4]. The leniency on what platforms can be used by practices for telehealth visits will also likely change after the pandemic. These possible changes will likely be rolled out at different times for different carriers so it will be critical to review EOBs and look at insurers' websites and newsletters.

It will be important to learn the codes and understand what codes different insurers require in order to be properly reimbursed for your work. Remembering to get consent for visits, to document what was done, to adhere to procedures that are medically necessary, and to code correctly will help practices receive payment for these services. It would be helpful to medicine in general if the commercial insurance companies and CMS provided a uniform approach and guideline for telemedicine coding. Until such time that these stakeholders provide a consistent and uniform coding guide to telemedicine, remember that "it depends" as you select the appropriate code, modifier, and place of service for telemedicine encounters.

\section{Compliance with Ethics Guidelines}

Conflict of Interest The authors declare no conflicts of interest relevant to this manuscript.

Human and Animal Rights and Informed Consent This article does not contain any studies with human or animal subjects performed by any of the authors.

\section{References}

1. AMA Telehealth Quick Guide https://www.ama-assn.org/practicemanagement/digital/ama-telehealth-quick-guide. Accessed 22 Jul 2020

2. American Medical Association: CPT 2020 Professional Edition. 5th ed., revised Chicago (IL) AMA, 2019.

3. Billing \& Coding by Type of Visit https://www.acponline.org/ practice-resources/business-resources/telehealth/physician-videovisits Accessed 22 Jul 2020.

4. Telehealth Services. https://www.cms.gov/Outreach-and-Education/ Medicare-Learning-Network-MLN/MLNProducts/Downloads/ TelehealthSrvcsfctsht.pdf Accessed 22 Jul 2020.

Publisher's Note Springer Nature remains neutral with regard to jurisdictional claims in published maps and institutional affiliations. 